\title{
Multimodal Oncological Therapy Comprising Stents, Brachytherapy, and Regional Chemotherapy for Cholangiocarcinoma
}

\author{
Tomáš Andrašina*, Vlastimil Válek*, Jiří Pánek*, Zdeněk Kala ${ }^{\dagger}$, Igor Kiss ${ }^{\ddagger}$, Štěpán Tuček ${ }^{\ddagger}$, and Pavel Šlampa ${ }^{\S}$ \\ Departments of ${ }^{*}$ Radiology and ${ }^{\dagger}$ Surgery, University Hospital Brno, Masaryk University, Deptartments of ${ }^{\dagger}$ Comprehensive Oncology Care, \\ and ${ }^{\S}$ Radiotherapy, Masaryk Memorial Cancer Institute, Masaryk University, Brno, Czech Republic
}

Background/Aims: To prospectively evaluate our palliative management of unresectable cholangiocarcinoma (CC) treated with tailored multimodal oncological therapy. Methods: Between January 2005 and January 2010, 50 consecutive patients with unresectable CC and jaundice were palliated with percutaneous drainage. Forty-three patients underwent metallic-stent implantation followed by brachytherapy. Patients were divided into two arms: the intra-arterial chemotherapy arm (IA arm, $n=17$ ) consisted of patients treated with locoregional treatment (IA admission of Cisplatin and 5-fluorouracil, or chemoembolization with Lipiodol) and/or systemic chemotherapy, while the systemic chemotherapy arm (IV arm, $n=23$ ) included all the other patients, who were treated only with systemic chemotherapy. Results: In total, 78 metal self-expandable stents were placed. Hilar involvement with mass-forming and periductal infiltrating types of CC (84\%) was predominant. The average number of percutaneous interventional procedures was 11.61 per patient (range, 4-35). The median overall survival from diagnosis of disease for all patients was 13.5 months (range, 11.0-18.8 months). The median overall survival times were 25.2 months (range, 15.2-31.3 months) and 11.5 months (range, 8.5-12.6 months) in the IA and IV arms, respectively $(p<0.05)$. The 1-, 2-, and 3-year survival rates in the IA and IV arms were $88.2 \%, 52.9 \%$, and $10.1 \%$ and $43.5 \%, 25.4$, and $0 \%$, respectively. There were no major complications (WHO III/IV) due to interventional procedures. Conclusions: We could reach acceptable prognosis in patients with unresectable CC using complex tailored oncological therapy. However, the main limitations of prolonging survival are performance status, patient compliance and the maintaining of biliary tract patency. (Gut Liver 2010;4(Suppl. 1):S82-88)

Key Words: Cholangiocarcinoma; Brachytherapy; Infusions; Intra-arterial; Stents

\section{INTRODUCTION}

The most common symptom of cholangiocarcinoma is painless jaundice. Even though the cause of jaundice could be a small-size tumor, the unfavorable localisation of the common perihilar type greatly influences the prognosis of patients. In intrahepatic-peripheral cholangiocarcinoma, jaundice is frequently missing and we may observe masses which are primarily very problematic to overcome by surgical treatment. Perihilar tumor extension is sometimes hard to be diagnosed preoperatively by using magnetic resonance cholangiopancreatography, multidetector computed tomography (with use of MinIP projections) or direct cholangiography. Nevertheless, preoperative imaging can play a crucial role in the planning of surgical or palliative procedures.

A variety of surgical procedures exist, according to the localization of cholangiocarcinoma. As to intrahepatic cholangiocarcinoma there is no difference in resections from other intrahepatic malignancies. In extrahepatic bile duct cancer for selective cases of localized tumors of middle choledochus or high-risk patients, it is possible to perform limited bile duct resections. In cases of late-stage disease, surgical procedures can comprise hemipancreatoduodenectomy which is a preferable treatment for middle and lower bile duct cancers, extended hepatectomies, trisectoriectomies with or without caudate lobectomies, lym-

\footnotetext{
Correspondence to: Vlastimil Válek

Department of Radiology, University Hospital Brno, Masaryk University Brno, Jihlavská 340/20, 62500 Brno, Czech Republic

Tel: +420-532-233-007, Fax: +420-532-233-009, E-mail: v.valek@fnbrno.cz
}

DOI: $10.5009 /$ gnl.2010.4.S1.S82 


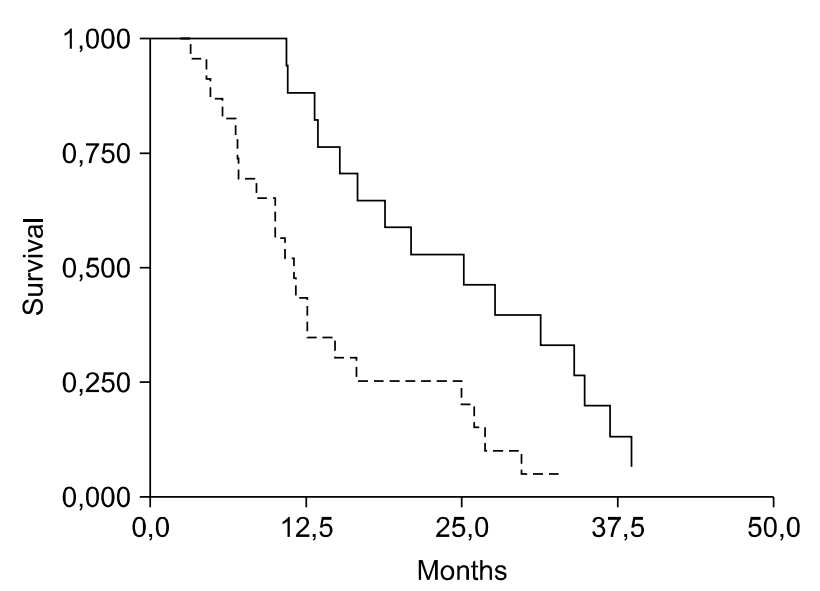

Fig. 1. Kaplan-Meier overall survival curves in the IV arm (dotted line) and the IA arm (solid line).

phadenectomy for hilar bile duct carcinoma. Strategy for surgical treatment depends not only on the localization and extension of disease but also on morphological characteristics of cholangiocarcinoma. As to the intraductal type (papillary and nodular types), superficial spreading is typical and it can exceed the macroscopic margin by 20 $\mathrm{mm}$. Different from this are the infiltrating and mass-forming types, in which submucosal spreading is typical (direct, lymphatic, perineural invasion) within a distance of $10 \mathrm{~mm}$ from the observable tumor. ${ }^{1}$

Understanding the pathologic characteristics of each type of tumor can be helpful in developing a differential diagnosis and in treatment planning. ${ }^{2}$ This statement is not only valid for surgical treatment but also for palliative procedures where multiple approaches are being used in cholangiocarcinoma. The intrahepatic mass-forming type of cholangiocarcinomas as well as other hepatic malignancies can be succesfully palliated by use of radiofrequency or microwave ablation. Intra-arterial chemotherapy, chemoembolization or a combination of these treatments can be applied in tumors in which local ablative therapies are not sufficient, cannot be used, and tumor shows high arterial enhancement. As to cholangiocarcinomas affecting the central bile ducts (mass-forming and periductal infiltrating types) the key issue is to achieve accurate bile duct patency.

Bile duct obstruction, if not adequately compensated, can result in intrahepatic cholestasis with significant reduction of the patient's quality and length of life. The most frequent localization for the infiltrating type of cholangiocarcinoma is proximal or middle bile duct, where the use of percutaneous approach of prothesis implantation is preferable compared to endoscopic route. According to our experience and to published studies, ${ }^{3,4}$ the pa-

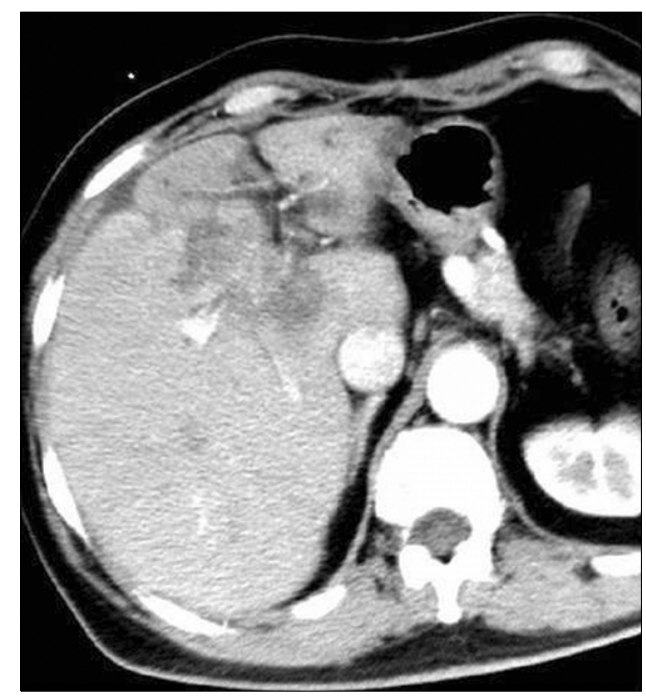

Fig. 2. Female (67 years old) with histologically confirmed cholangiocarcinoma in which radical resection was not possible. Dilatation of intrahepatic biliary ducts is in segments (CT performed in October 2005).

tency of metal stents lasts longer in comparison with plastic drains. Moreover, tolerance and patient compliance are higher when using a metallic stent.

External beam radiotherapy and mainly intraluminal brachytherapy can extend stent patency and therefore the quality and survival of patients. ${ }^{5,6}$ The advantage of brachytherapy with $192 \mathrm{Ir}$ is the possibility of delivering higher doses of radiation, in a short time, without significant irradiation of normal tissues. Between the years 1997-2000 we showed the benefit of brachytherapy in prospective randomized study. In the group of patients treated with combined radiotherapy and with stent implantation the mean survival was 388 days. In the group of patients treated only with stent insertion the mean survival was 298 days. $^{7}$ The use of intraluminal brachytherapy following the implantation of metallic self-expandable stent became the standard method in our institute.

Systemic chemotherapy for a long time showed only limited benefit for patients with cholangiocarcinoma, with only a $30 \%$ response rate being achieved. ${ }^{8}$ The combination of systemic chemotherapy (gemcitabine) and regional chemoembolizations may enhance the effect of palliation in a selected group of patients with a 21.1-23 months (1, 2 , and 3-year survival rates $51.3 \%, 27.5 \%$, and $27.5 \%$, respectively) reported median of overall survival. ${ }^{9-11}$ The potential benefit of regional chemotherapy with port system implanted percutaneously in intrahepatic cholangiocarcinoma (CC) was also reported by Tanaka et al. ${ }^{12}$ with a 26-month mean overall survival. All in all, intra-arterial 


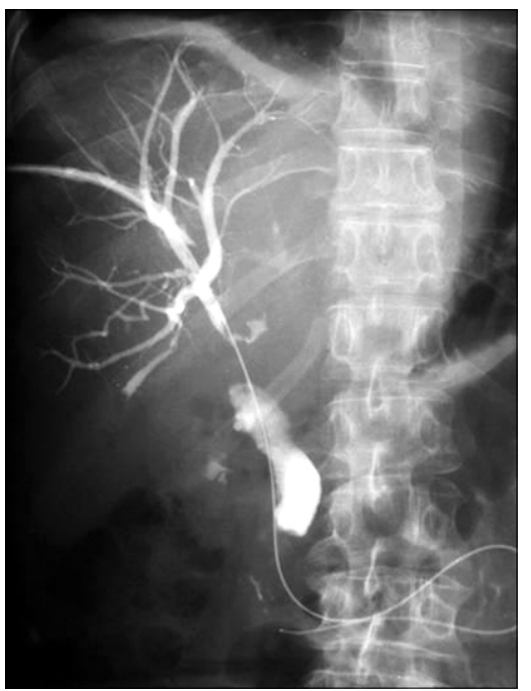

Fig. 3. A percutaneous biliary drain (PTD) is inserted after percutaneous cholangiography (PTD performed in October 2005).

therapies (chemoembolizations and chemoperfusions) offer a potential benefit and prolonged survival for patients with unresectable cholangiocarcinoma.

\section{MATERIALS AND METHODS}

Between January 2005 and January 2010, a total of 465 patients were admitted to our institution for the treatment of jaundice by percutaneous drainage. In some of them, the endoscopical approach was not successful or was not primarily indicated because of the location, the character of disease or the need for further oncological management. A malignant etiology of stricture was recognized in 318 patients $(68.4 \%$ of all patients, of which $54 \%$ female). With 50 patients $(15.7 \%)$, cholangiocarcinoma was the second most common malignancy causing biliary stricture (44\% were pancreatic adenocarcinomas, $15 \%$ were liver metastases [variety of primary tumors], $10 \%$ were gall bladder neoplasms).

Before percutaneous dranaige, all patients underwent abdominal CT or MRI scan to optimize percutaneous route. Histological verification of stricture, managed by transhepatic transluminal forceps biopsy, was mandatory after successful drainage procedure, whenever preprocedural surgery or endoscopy had not previously confirmed the diagnosis.

Every patient was presented to the Oncological Indication Committee - a multidisciplinary board of specialists consisting of oncologists, surgeons, radiologists, hepatologist, radiotherapeutist, pathologist - informed consent was obtained and the Institute Ethics Committee also gave

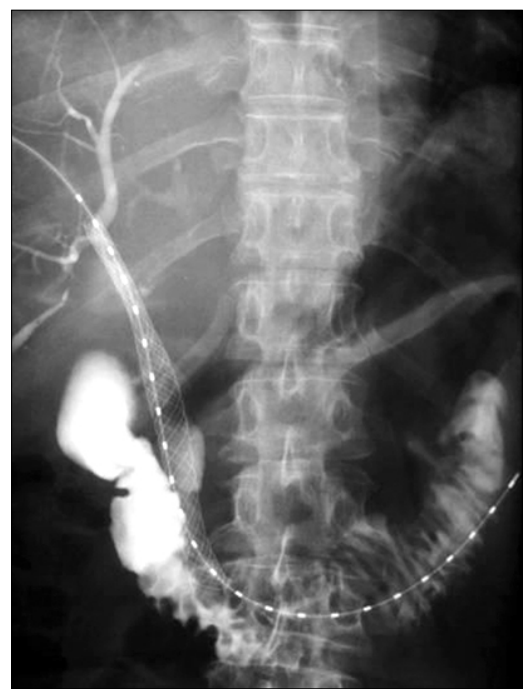

Fig. 4. Self-expandable metal stent is implanted after successful drainage, through which a measuring wire is inserted for the subsequent performance of brachytherapy (in November 2005).

their approval to the study.

A standardized therapeutic plan was created for every patient with cholangiocarcinoma. The main factors influencing the extent of the therapeutic plan was performance status, patient compliance, and the stage of disease.

Inclusion criteria for prospective study of multimodal therapy were: unresectable disease, Karnofsky's Perfomance Score of $80 \%$ and above, functional percutaneous drain (8-10 F) inserted for an average period of 10-14 days with subsequent decrease of bilirubine levels and other liver function tests, no extrahepatic metastases.

The full multimodality treatment plan consisted of metallic uncovered self-expandable stent insertion (the number of stents depended on the anatomy and extent of the disease), intraluminal brachytherapy and locoregional chemotherapy.

Biliary stent had to be long enough to cover the proximal and distal segments of the stricture and the application of multiple stents in a Y-shaped pattern was predominant. Later on, the patient underwent brachytherapy daily for 2-5 consecutive days in total dose of 12-30 Gy in 2 to 5 fractions. The stent was secured by a $4-\mathrm{F}$ safety catheter after brachytherapy. After an uncomplicated set of biliary interventions, the patient was prepared for regional chemotherapy. The patient was equipped with a percutaneously implanted port catheter, particularly if he/she suffered from multiple, poorly vascularized foci of cholangiocarcinoma. Cisplatin and 5-Fluorouracil were the administered chemotherapeutic agents. (Cisplatin, dose of $45 \mathrm{mg} / \mathrm{m}^{2}$ via IA infusion lasting 1 hour on D1 only; 5-Fluorouracil, dose of $450 \mathrm{mg} / \mathrm{m}^{2}$ via 


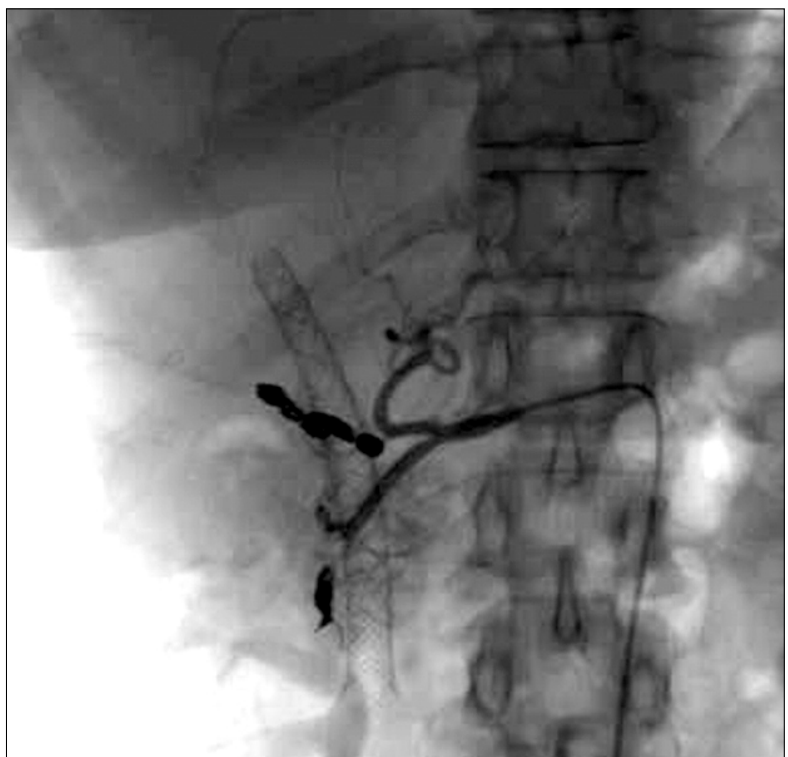

Fig. 5. Port catheter is percutaneously inserted into the hepatic artery for regional chemotherapy (in November 2005).

1-hour IA infusion on D1-5, $20 \mathrm{mg} / \mathrm{m}^{2}$ of Leucovorin was administered before 5-Fluorouracil). A less frequent application of intra-arterial chemotherapy (suitable for vascularized tumors) consisted of a time-limited catheter insertion, optimally 1-3 liver segments. It usually lasted 3-5 days and was completed by a non-selective chemical embolization with Lipiodol. Periprocedural intravenous antibiotics were used to prevent hepatic abscess formation.

Systemic chemotherapy included the continuous IV infusion of 5-Fluorouracil $\left(900 \mathrm{mg} / \mathrm{m}^{2}\right.$ during the first four days) in combination with leucovorin $\left(20 \mathrm{mg} / \mathrm{m}^{2}\right)$.

Statistical analysis (Kaplan-Meier life-table analysis, estimated cumulative survival and curves) was generated with the aid of NCSS statistical software (NCSS Systems, Kayesville, UT, USA). A 0.05 p-value was taken as statistical significant and the groups of patients were compared according to the log-rank test.

\section{RESULTS}

Out of 50 patients diagnosed with cholangiocarcinoma, 7 (median age, 79.5) were excluded from the study after initial percutaneous drainage, since they did not meet the inclusion criteria. The main reason was a Karnofsky Performance Score below $80 \%(n=5)$, disagreement with recommended procedures $(n=1)$, disseminated disease metastases to lung and liver $(n=1)$.

A total of 78 metal self-expandable stents was successfully placed to malignant biliary stenosis for 43 patients (1.81 stent per patient). Hilar involvement was predom-

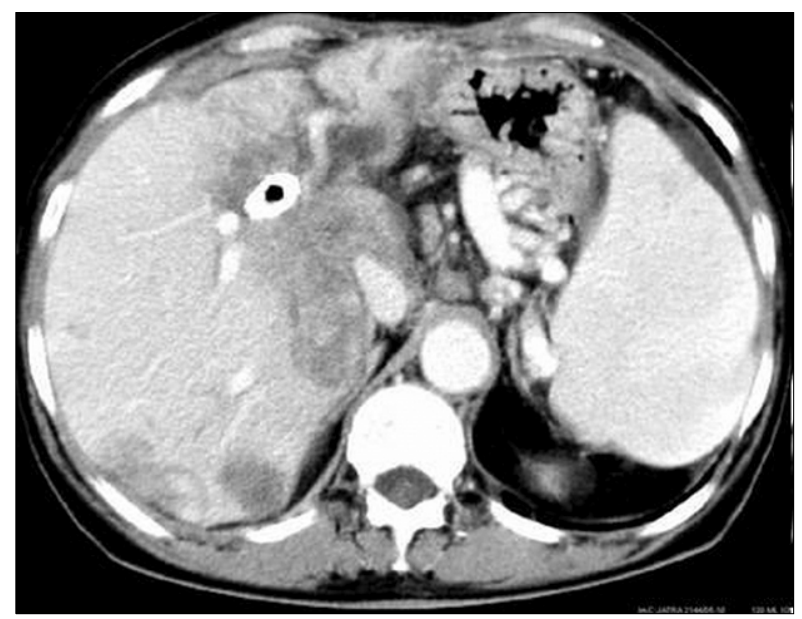

Fig. 6. CT performed at 1 month before the patient died. Local progression and distant metastases were evident (in August 2008).

inant (38 patients, 88\%), mass-forming and periductal infiltrating types, only 2 patients with polypoid mass, 4 patients $(9 \%)$ were diagnosed with intrahepatic CC, and implantation of stent was necessary due to hilar progression. Only 1 patient had unresectable tumor of distal choledochus.

Median time from the first percutaneous intervention to the successful implantation of metal stent was 44 days, and the average number of percutaneous sessions totalled 5.2 (2-12). This depended on the favorable effect of percutaneous drainage (for at least 2 weeks, with substantial improvement of laboratory tests), of the technique and the necessary time for histological confirmation of malignant stenosis.

Selection of patients for the implementation of intra-arterial chemotherapy was based on preprocedural studies of contrast-enhanced imaging techniques (CECT, CE-MRI, CEUS). A patient was assigned to the IA arm whenever lesion also showed hypervascularity of some of the area of cholangiocarcinoma, and there was no anatomical variation restraining intra-arterial procedure. There were 17 patients selected for the IA arm. Patients who were not suitable for IA chemotherapy, received only systemic chemotherapy (IV arm, $n=23$ ). Three patients were excluded from the study for rapid deterioration after stent implantation and brachytherapy, which prevented any further use of chemotherapy (overall survival, 2.77-4.87 months). At the end of the study, 4 patients were still alive ( 2 in IA arm, 2 in IV arm).

Average age of patients in time of diagnosis was 57.22 (range, 35-74) in IA arm and 60.43 (range, 45-71) in IV arm. The median overall survival from diagnosis of dis- 
Table 1. Overall Survival and Statistics

\begin{tabular}{lcccc}
\hline & $\mathrm{n}$ & $\begin{array}{c}\text { Overall } \\
\text { survival, mo }\end{array}$ & $\begin{array}{c}\text { Lower } \\
95 \% \text { C.L. }\end{array}$ & $\begin{array}{c}\text { Upper } \\
95 \% \text { C.L. }\end{array}$ \\
\hline IV arm & 23 & 11.50 & 8.50 & 12.60 \\
IA arm & 17 & 25.20 & 15.20 & 31.30 \\
Age & 43 & 58 years & & \\
Biliary & 43 & 11.61 & (range, 4-35) & \\
$\quad$ interventions & & & (range, 1-16) & \\
$\quad \begin{array}{l}\text { Intra-arterial } \\
\quad \text { interventions }\end{array}$ & 17 & 3 & & \\
\hline
\end{tabular}

ease for all patients selected for IA arm or IV arm was 13.5 months (range, 11.0-18.8 months). In IA arm, the median overall survival was 25.2 months (range, 15.2-31.3 months), and in IV arm 11.5 months (range, 8.5-12.6 months), which shows statistical difference $p$ $<0.05$. Median overall survival, 1,2 and 3 year survival rates are described in Tables 1 and 2 .

The average number of percutaneous interventional procedures were 11.61 per patient (range, 4-35). We did not notice any major complications (WHO III/IV) due to interventional procedures. Minor complications of biliary interventions were observed in $7.3 \%$ on the basis of per-patient analysis, and the most frequent was hemobilia and subcapsular hematoma. Forty-three patients did not show any direct complications after brachytherapy. Nausea, fever or mild abdominal pain were noticed in most patients, and were probably caused by percutaneous drainage. No specific data for adverse events in the course of systemic chemotherapy were collected. In IA chemotherapy, most common was post-embolization syndrome in patients where Lipiodol was administered (referred by oncologists in $80 \%$ of patients, who required antiemetic therapy).

Median duration of stent patency was 5.75 months (average, 7.29 months) in patients where subsequent redrainage was necessary $(n=14)$. Stent patency in the whole group (endpoint of stent patency = time of death or recognized obstruction) was 6.23 in median (10.15 in average). 7 out of the 10 patients surviving more than 25 months in the study (8 patients in IA arm and 2 in IV arm) had to undergo redrainage procedure which resulted in the implantation of an additional stent or a permanent external/internal drainage.

\section{DISCUSSION}

A complete resection remains the only therapy that offers the possibility of long-term survival or curative treatment for Klatskin tumor. ${ }^{13,14}$ A 5-year survival rate can
Table 2. Survival at Specific Event Times

\begin{tabular}{lcccr}
\hline & $\begin{array}{c}\text { Event } \\
\text { time, yr }\end{array}$ & $\begin{array}{c}\text { Cumulative } \\
\text { survival }\end{array}$ & $\begin{array}{c}\text { Lower } \\
\text { 95\% C.L. }\end{array}$ & $\begin{array}{c}\text { Upper } \\
95 \% \text { C.L. }\end{array}$ \\
\hline IV arm & 1 & 43.48 & 23.22 & 63.74 \\
IA arm & 1 & 88.24 & 72.92 & 100.00 \\
IV arm & 2 & 25.36 & 7.25 & 43.47 \\
IA arm & 2 & 52.94 & 29.21 & 76.67 \\
IV arm & 3 & 0.00 & 0.00 & 0.00 \\
IA arm & 3 & 19.85 & 0.00 & 39.72 \\
\hline
\end{tabular}

reach up to $22-36 \%$ for patients with resected intrahepatic cholangiocarcinoma. However, resection with a curative intent is performed only in $19-46 \%$ of patients and the majority of patients are referred to palliative procedures. $^{15}$

Palliative procedures should relieve the symptoms, improve quality of life, and finally prolong overall survival. However, palliative approaches for hepatobiliary malignancies are variable, ranging from best supportive care to combination of chemotherapy, radiotherapy, photodynamic therapy, embolizations with particles. Median survival time of palliated patients varied greatly from 3 to 29.3 months. $^{16,17}$

The aim of this prospective study is to prove how complex therapy can improve survival of patients. There are some limitations which may have decreased the statistical strength of results preferring IA therapy. This study was not randomized and patients were selected according to the principle of individually tailored multimodal oncological therapy. The same approach was then applied to patients able to undergo IA chemotherapy. In 2 patients from IA arm (17 patients) tumor was recognized with intraluminal growth pattern by radiological studies (histological diagnosis refers to adenocarcinoma). Intraluminal growth pattern most probably corresponds to papillary subtype, which has late transmural extension as this subtype is associated with more favorable prognosis. ${ }^{18}$ Similarly, the more vascular or hypervascular tumors (which could be the target of chemoembolizations) have naturally better prognosis than hypovascular tumors, well-differentiated adenocarcinomas are more frequent in hypervascularized tumors than hypovascularized, where multiple tumors were noticed more frequently. ${ }^{19}$

In addition to what can be observed about the median survival, another remarkable fact is the minimal survival of patients with complex therapy, reaching 11 months. Patients underwent a high number of biliary interventions (average, 11.61) to reach sufficient biliary drainage, but we suggest that careful maintaining of patency of biliary tract can prolong patient's survival. In order for this to 
happen, there has to be a very good compliance and tolerance of the patient. Long-standing biliary obstruction alone can cause moderate atrophy of liver lobes, ${ }^{20}$ which deteriorates the patient functional parenchyma of liver and thus shortening time to hepatorenal failure.

We suggest several ways to increase survival of patients in the future. Promising results were published according to use of drug-eluting beads for intrahepatic $\mathrm{CC}^{21}$ Currently, chemoembolization with drug-eluting beads replaced chemoembolizations with Lipiodol in our institute. Rationales for this are in characteristics of Lipiodol, which shows quicker washout from lesions of CC as compared to hepatocellular carcinoma and chemotherapeutics are diluted from Lipiodol in several hours compared to the prolonged diffusion from drug-eluting beads. New advancements in systemic therapies can be represented by using targeted therapy (Sorafenib) which in laboratory conditions can potentially suppress the growth of cholangiocarcinoma, and alone or in combination therapy may provide a promising rationale for future use. Synergistic effects were observed when Sorafenib was combined with Doxorubicin (frequently used agent in drug-eluting beads). ${ }^{22}$

Improving the prognosis of patients with modern chemotherapeutic agents and palliations causes a problem with metal stent obstruction. An obstruction of metal stent may occur due to intimal hyperplasia and tumor ingrowth or it can also be obstructed by cellular detritus. Management of stent obstruction resulted in a significant increase in the number of nonvascular interventions for patients and also increased the cost of palliation of the disease. In our ongoing prospective study we are trying to analyze the benefit of bipolar intraluminal radiofrequency ablations before a stent insertion or whenever a stent occlusion occurs.

In conclusion, we showed favorable prognosis for patients with unresectable cholangiocarcinoma using complex tailored oncological therapy. In future trials it is necessary not only to count on diagnosis and localizations of cholangiocarcinoma but also on morphological characteristics (growth pattern, vascularization) which can greatly influence the prognosis of the studied groups. In Central Europe, the greatest limitation to the elaboration of accurate and prospective randomized studies of palliation is the low incidence of cholangiocarcinoma as well as the advantages of a surgical approach.

\section{REFERENCES}

1. Seyama Y, Makuuchi M. Current surgical treatment for bile duct cancer. World J Gastroenterol 2007;13:1505-
1515.

2. Chung YE, Kim MJ, Park YN, et al. Varying appearances of cholangiocarcinoma: radiologic-pathologic correlation. Radiographics 2009;29:683-700.

3. Kaassis M, Boyer J, Dumas R, et al. Plastic or metal stents for malignant stricture of the common bile duct? Results of a randomized prospective study. Gastrointest Endosc 2003;57:178-182.

4. Lammer J, Hausegger KA, Fluckiger F, et al. Common bile duct obstruction due to malignancy: treatment with plastic versus metal stents. Radiology 1996;201:167-172.

5. Golfieri R, Giampalma E, Renzulli M, et al. Unresectable hilar cholangiocarcinoma: multimodality approach with percutaneous treatment associated with radiotherapy and chemotherapy. In Vivo 2006;20:757-760.

6. Eschelman DJ, Shapiro MJ, Bonn J, et al. Malignant biliary duct obstruction: long-term experience with Gianturco stents and combined-modality radiation therapy. Radiology 1996;200:717-724.

7. Valek V, Kysela P, Kala Z, Kiss I, Tomasek J, Petera J. Brachytherapy and percutaneous stenting in the treatment of cholangiocarcinoma: a prospective randomised study. Eur J Radiol 2007;62:175-179.

8. Kubicka S, Rudolph KL, Tietze MK, Lorenz M, Manns M. Phase II study of systemic gemcitabine chemotherapy for advanced unresectable hepatobiliary carcinomas. Hepatogastroenterology 2001;48:783-789.

9. Kirchhoff T, Zender L, Merkesdal S, et al. Initial experience from a combination of systemic and regional chemotherapy in the treatment of patients with nonresectable cholangiocellular carcinoma in the liver. World J Gastroenterol 2005;11:1091-1095.

10. Burger I, Hong K, Schulick R, et al. Transcatheter arterial chemoembolization in unresectable cholangiocarcinoma: initial experience in a single institution. J Vasc Interv Radiol 2005; 16:353-361.

11. Herber S, Otto G, Schneider J, et al. Transarterial chemoembolization (TACE) for inoperable intrahepatic cholangiocarcinoma. Cardiovasc Intervent Radiol 2007;30:1156-1165.

12. Tanaka N, Yamakado K, Nakatsuka A, Fujii A, Matsumura $\mathrm{K}$, Takeda K. Arterial chemoinfusion therapy through an implanted port system for patients with unresectable intrahepatic cholangiocarcinoma--initial experience. Eur J Radiol 2002;41:42-48.

13. Jarnagin WR, Fong Y, DeMatteo RP, et al. Staging, resectability, and outcome in 225 patients with hilar cholangiocarcinoma. Ann Surg 2001;234:507-517.

14. Chan SY, Poon RT, Ng KK, Liu CL, Chan RT, Fan ST. Long-term survival after intraluminal brachytherapy for inoperable hilar cholangiocarcinoma: a case report. World J Gastroenterol 2005;11:3161-3164.

15. Lee KH, Lee DY, Kim KW. Biliary intervention for cholangiocarcinoma. Abdom Imaging 2004;29:581-589.

16. Forsmo HM, Horn A, Viste A, Hoem D, Ovrebo K. Survival and an overview of decision-making in patients with cholangiocarcinoma. Hepatobiliary Pancreat Dis Int 2008;7:412-417.

17. Singal AG, Rakoski MO, Salgia R, et al. The clinical presentation and prognostic factors for intrahepatic and extrahepatic cholangiocarcinoma in a tertiary care centre. Aliment Pharmacol Ther 2010;31:625-633. 
18. Jarnagin WR, Bowne W, Klimstra DS, et al. Papillary phenotype confers improved survival after resection of hilar cholangiocarcinoma. Ann Surg 2005;241:703-712.

19. Nanashima A, Sumida Y, Abo T, et al. Relationship between pattern of tumor enhancement and clinicopathologic characteristics in intrahepatic cholangiocarcinoma. J Surg Oncol 2008;98:535-539.

20. Ito F, Cho CS, Rikkers LF, Weber SM. Hilar cholangiocarcinoma: current management. Ann Surg 2009;250:
210-218.

21. Aliberti C, Benea G, Tilli M, Fiorentini G. Chemoembolization (TACE) of unresectable intrahepatic cholangiocarcinoma with slow-release doxorubicin-eluting beads: preliminary results. Cardiovasc Intervent Radiol 2008;31:883-888.

22. Huether A, Hopfner M, Baradari V, Schuppan D, Scherubl H. Sorafenib alone or as combination therapy for growth control of cholangiocarcinoma. Biochem Pharmacol 2007; 73:1308-1317. 